\title{
Deriving fundamental parameters of millisecond pulsars via AIC in white dwarfs
}

\author{
A. Taani ${ }^{1 \dagger}$, C. M. Zhang ${ }^{1}$, Y. H. Zhao ${ }^{1}$ and A. Moraghan ${ }^{2}$ \\ ${ }^{1}$ National Astronomical Observatories, Chinese Academy of Sciences, Beijing 100012, China \\ †email: alitaani@bao.ac.cn \\ ${ }^{2}$ Center for Galaxy Evolution Research and Department of Astronomy, Yonsei University, \\ Seoul 120-749, Republic of Korea
}

\begin{abstract}
We present a study of the observational properties of Millisecond Pulsars (MSPs) by way of their magnetic fields, spin periods and masses. These measurements are derived through the scenario of Accretion Induced Collapse (AIC) of white dwarfs (WDs) in stellar binary systems, in order to provide a greater understanding of the characteristics of MSP populations. In addition, we demonstrate a strong evolutionary connection between neutron stars and WDs with binary companions from a stellar binary evolution perspective via the AIC process.
\end{abstract}

Keywords. Neutron stars, white dwarfs, cataclysmic variables, fundamental parameters.

\section{Introduction}

Observable parameters of binary Millisecond Pulsars (MSPs), e.g. mass of the pulsar, mass of the companion, spin period, orbital period, eccentricity, etc., are used to probe the past accretion history of the MSPs. The purpose of this proceeding is to demonstrate how to infer some of the observable quantities (spin period, magnetic field and mass) during the Accretion Induced Collapse (AIC) of a white dwarf (WD) on its way to become a member of the MSP family.

\section{Evolution of spin period}

We are able to determine the spin period of MSPs originating from WDs. The process begins with simple Keplerian frequency. We assume that the angular velocity of the NS is equal to the Keplerian angular velocity, $v_{K}$, of the magnetosphere, at roughly the Alfvén surface,

$$
v_{K} \propto R_{N S}^{-3 / 2} \rightarrow P_{M S P} \sim R_{N S}^{3 / 2}
$$

from which we obtain the spin of the MSP, $P_{M S P}$, as a function of the WD spin, $P_{W D}$.

$$
P_{M S P} \sim P_{W D, \min }\left(\frac{R_{N S}}{R_{W D}}\right)^{3 / 2}
$$

where $P_{W D, \text { min }}$ is the minimum spin period of the standard WD (Warner 1995). Assuming $P_{W D, \text { min }} \sim 30 \mathrm{~s}, R_{N S}=10 \mathrm{~km}$ and $R_{W D}=1000 \mathrm{~km}$, we thus obtain,

$P_{M S P} \sim 1 \mathrm{~ms}$. Fig. 1 (left) shows the observed and derived spin period distributions of MSPs. As for the observed MSPs, they show a relatively Gaussian distribution. According to these distributions, the ratio of MSPs originating from CVs is about $\sim 10 \%$. This result agrees with some theoretical predictions such as those by Warner (1995) and Warner \& Woudt (2002).

\section{Magnetic field}

To investigate the correlation between the magnetic fields of MSPs with bottom fields of CVs (where the field is partially restructured due to accretion), we follow the model 

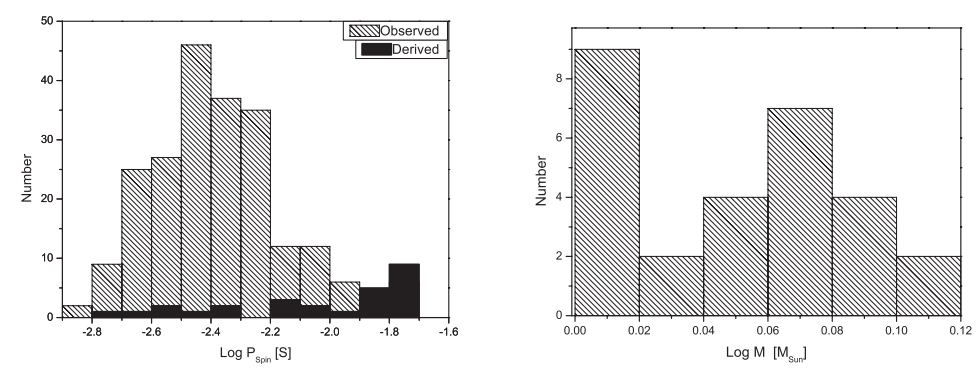

Figure 1. Left: The distribution of MSPs in terms of spin-period (observed data from Manchester et al.(2005). Right: Mass distribution of massive CVs (Ritter \& Kolb 2011).

of Zhang et al. (2009). The magnetic flux is assumed to be conserved. This corresponds to the magnetic fields produced by AIC.

$$
B_{N S}=B_{f, W D} \times\left(\frac{R_{W D}}{R_{N S}}\right)^{2}
$$

If we adopt $R_{N S}=15 \times 10^{5} \mathrm{~cm}$ and $B_{f, W D} \sim 10^{3} \mathrm{G}$ in $\mathrm{CVs}$, the minimum value, $B_{N S} \sim 10^{8.5-9} G$.

\section{Mass}

The sample of CVs whose masses we have considered is the set of binary systems collected by Ritter \& Kolb (2011). Among them we have 26 massive CVs in the range $1.0-1.3 \mathrm{M}_{\odot}$. Fig. 1 (right) shows the relatively Gaussian distribution, with mean at $\mathrm{M}_{\mathrm{CV}} \sim 1.1 \mathrm{M}_{\odot}$. A summary of the known properties of these systems is given in Table 1 of Taani et al. (2012). Note that the AIC process leads to a MSP with mass less than Chandrasekhar limit Zhang et al. (2011). This provides evidence for the AIC in massive CVs and evolutionary hypotheses of MSP birthrate.

\section{Summary and Conclusions}

(a) CVs would be invoked via the capability of producing a significant portion of the MSPs via the AIC process, a regime which may be unattainable by normal channels.

(b) We find that the quantitative implications of our calculations are that we estimate the expected $P$ in the observed of MSPs which could have originated from CVs to be $\sim 10 \%$.

(c) We further find that the predictions of some parameters after AIC process for the average levels are consistent with the observed MSP population. Future work will consider other quantities e.g. orbital period, eccentricity, and mass ratio (q) using more data sets from SDSS.

We are grateful for the discussion with Cole Miller. This research has been supported by NBRPC (2009CB824800, 2012CB821800) and the NSFC (10773017, 11173034).

\section{References}

Manchester, R. N., Hobbs, G. B., Teoh, A., et al. 2005 AJ 129, 1993

Ritter, H. \& Kolb, U., 2011, VizieR Online Data Catalog 1, 2018

Taani, A., Zhang, C. M., Al-Wardat, M., et al. 2012 ApESSS 340, 147

Warner, B., 1995, Cataclysmic Variable Stars. Cambridge Astrophysics Series vol. 28

Warner, B. \& Woudt, P. A., 2002 The Physics of Cataclysmic Variables and Related Objects. Astronomical Society of the Pacific Conference Series vol. 261

Zhang, C. M., Wickramasinghe, D. T., Ferrario, L., et al. 2009 MNRAS 397, 2208

Zhang, C. M., Wang, J., Zhao, Y. H., et al. 2011 A\&A 527, 83 